\title{
Disfunção Erétil em Homens com Esclerose Sistêmica ${ }^{(*)}$
}

\section{Erectile Dysfunction in Systemic Sclerosis}

\author{
João Francisco Marques Neto ${ }^{(1)}$, Gláucio R. Werner de Castro ${ }^{(2)}$, \\ Percival D. Sampaio-Barros ${ }^{(3)}$ e Adil M. Samara ${ }^{(1)}$
}

\section{RESUMO}

O objetivo deste trabalho é analisar a prevalência da disfunção erétil (DE) e a utilidade do Índice Internacional de Função Erétil (IIFE) na investigação da DE em pacientes com esclerose sistêmica (ES). Quinze homens com ES foram questionados acerca dos sintomas da DE e submetidos ao questionário de IIFE. Seus prontuários foram revisados para identificar outras possíveis causas da DE. Os pacientes estudados eram predominantemente caucasóides e tinham ES difusa. DE foi referida por $67 \%$ dos pacientes, iniciando-se, em média, 29 meses após o início da ES; quatro pacientes relataram início concomitante da DE e da ES. O diagnóstico da DE pelo IIFE e por interrogação direta foi concordante em todos os pacientes. Não foi encontrada associação nítida entre DE e extensão do envolvimento cutâneo, envolvimento visceral, drogas, escore cutâneo total e duração da doença. Cinco pacientes com DE também tinham outras causas de impotência. A DE afeta um grande porcentual de homens acometidos por esclerose sistêmica e o IIFE pode ser um instrumento válido em sua avaliação. A alta incidência da DE em jovens com ES e a eventual concomitância do início da ES e da DE enfatizam a necessidade de estudos posteriores sobre uma provável relação causal.

Palavras-chave: esclerose sistêmica, disfunção erétil, Índice Internacional de Função Erétil.

\begin{abstract}
The aim of this report is to analyze the incidence of erectile dysfunction (ED) and the usefulness of the International Index of Erectile Function (IIEF) on the investigation of ED in men with systemic sclerosis (SSc). Fifteen male SSc patients were asked about the presence of ED symptoms and were submitted to the IIEF questionnaire, after obtaining informed consent. Their medical data were reviewed in the search of other possible causes of ED. The studied patients were predominantly Caucasian and had diffuse SSC. $E D$ was referred by $67 \%$ of them, and it was first noted after an average of 29 months of SSc onset; four patients referred concomitant onset of ED and SSC. All men with ED detected by IIEF questionnaire also reported $E D$ when directly asked, and all men who affirmed having ED were also identified by IIEF. There were no clear association between ED and extent of cutaneous involvement, visceral involvement, drugs, total skin score and disease duration. Five patients with ED also had other causes of impotence. ED affects a high percentage of male SSc patients, and the IIEF may be a valid instrument in its evaluation. The high incidence of ED in young SSC patients and the occasional concomitance of the onset of SSC and $E D$ warrants further evaluation of a causal association.
\end{abstract}

Keywords: systemic sclerosis, erectile dysfunction, International Index of Erectile Function.

quaisquer fatores que afetem o corpo cavernoso, a irrigação peniana, a inervação do pênis ou os eixos hormonais. Drogas e distúrbios psicológicos também estão entre as principais causas da DE.

Queixas de sintomas da DE, apesar de comuns em homens com esclerose sistêmica (ES), são freqüentemente negligen-

A disfunção erétil (DE) é definida, atualmente, como a incapacidade de obter ou manter uma ereção suficiente para um desempenho sexual satisfatório ${ }^{(1)}$. A disfunção erétil é, freqüentemente, multifatorial; pode ser causada por

* Trabalho realizado na Disciplina de Reumatologia do Departamento de Clínica Médica da Universidade Estadual de Campinas (FCM-UNICAMP). Recebido em 08/12/03. Aprovado, após revisão, em 30/01/04

1. Professor Titular. FCM-UNICAMP.

2. Ex-Residente. FCM-UNICAMP.

3. Assistente-Doutor. FCM-UNICAMP

Endereço para correspondência: Prof. Dr. João Francisco Marques Neto. Disciplina de Reumatologia, Departamento de Clínica Médica. Faculdade de Ciências Médicas - Universidade Estadual de Campinas ( FCM-UNICAMP). Barão Geraldo, Campinas, SP. CEP 13081-970. E-mail: jfmn@directnet.com.br 
ciadas por seus médicos. Talvez por isso, embora seu diagnóstico não seja difícil, pouco se conhece sobre a incidência e as causas da DE na ES. O Índice Internacional de Função Erétil (IIFE) é um questionário breve, fidedigno e auto-aplicável, amplamente utilizado na avaliação da DE. Suas questões são divididas em cinco domínios: função erétil, função orgástica, desejo sexual, satisfação quanto ao intercurso e satisfação sexual global ${ }^{(2)}$. O domínio de função erétil do IIEF pode ser utilizado para o diagnóstico da DE, baseado nos critérios de Cappelleri ${ }^{(3)}$. O IIFE também pode ser utilizado na graduação dos sintomas da DE e na monitorizarão de suas respostas ao tratamento.

O presente estudo objetivou a avaliação da prevalência da DE e da utilidade do IIFE na investigação da disfunção erétil em homens com ES.

\section{MATERIAL E MÉTODOS}

Todo homem com ES seguido no Ambulatório de Esclerodermia do Hospital de Clínicas da Universidade Estadual de Campinas (Unicamp) foi interrogado quanto à presença de sintomas da $\mathrm{DE}$, e solicitados a responder a versão brasileira do IIFE $^{(4)}$. O diagnóstico de ES baseou-se nos critérios do Colégio Americano de Reumatologia ${ }^{(5)}$ e os prontuários dos pacientes foram revisados para identificar outras possíveis causas da DE.

O diagnóstico da DE baseou-se em questões específicas (Você tem alguma dificuldade em obter uma ereção? Você tem alguma dificuldade quando tem relações sexuais?) e, sempre que possível, nos critérios de Cappelleri para DE.

\section{RESULTADOS}

Quinze homens com ES acompanhados no Ambulatório de Esclerodermia de nossa instituição concordaram em responder ao questionário, após assinarem termo de consentimento informado. Os pacientes eram em sua maioria caucasóides (73.3\%), com média de idade de 45 anos (16 a 65 anos). Houve predomínio de envolvimento cutâneo difuso (66.7\% dos pacientes), com duração média da doença igual a 38 meses. A média do escore cutâneo (método de Rodnan modificado) foi 16 ( \pm 8). Fenômeno de Raynaud foi referido por $93,3 \%$ dos pacientes.

Quando questionados diretamente sobre a presença da DE, $66,7 \%$ dos pacientes deram respostas positivas. O início da DE deu-se, em média, 29 meses após os primeiros sintomas da ES; quatro pacientes referiram início concomitante da ES e da DE, e apenas um referiu sintomas da DE previamente ao início da ES. Oito pacientes não mantiveram relações sexuais nas quatro semanas antecedentes ao estudo, impossibilitando a aplicação dos critérios de Cappelleri; cinco destes pacientes não tinham parceiros sexuais no momento do estudo, mas três deles tinham e referiram não manter relações sexuais por medo de falharem, após várias tentativas mal-sucedidas.

Excluídos os homens que não mantiveram relações sexuais nas quatro semanas anteriores, as respostas ao IIFE resultaram em uma incidência da DE igual a $57.1 \%$, observados os critérios de Cappelleri. No entanto, as questões específicas permitiram diagnosticar DE em cinco dos oito pacientes que negaram atividade sexual recente. Todos os pacientes em que foi diagnosticada DE através do questionário do IIFE também relataram DE quando interrogados diretamente. Todos os homens que mantiveram relações sexuais recentes e que referiam DE quando questionados diretamente, também foram diagnosticados pelos critérios de Cappelleri.

A avaliação das questões individuais do IIFE revelou que $57,2 \%$ dos pacientes que mantiveram relações sexuais nas últimas quatro semanas não obtiveram ereções rígidas o suficiente para a penetração, enquanto apenas $28,6 \%$ relataram não ter dificuldades em manter a ereção até o final do intercurso. Entre todos os pacientes, apenas 33,3\% referiram como alta ou muito alta sua confiança em obter uma ereção e apenas 33,3\% relataram estar moderadamente ou muito satisfeitos com sua vida sexual global.

Comparados os pacientes com e sem DE, os primeiros tinham maior média de idade (51 anos versus 31 anos, diferença sem significância estatística), mas ambos os grupos abarcaram pacientes jovens e mais velhos (homens com DE: 23-65 anos; homens sem DE: 16-63 anos) (figura 1). Não foi observada associação estatística entre os sintomas da DE e a extensão do envolvimento cutâneo, envolvimento visceral, drogas e duração da doença (tabelas 1 a 3 ).

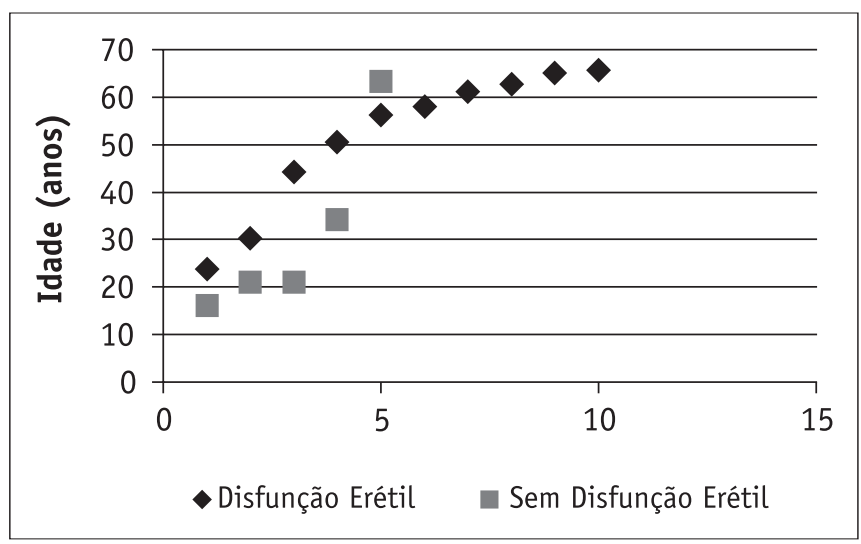

FiguRA 1 - Distribuição dos pacientes $(n=15)$, por idade. 
TABELA 1

Características ePidemiológicas

\begin{tabular}{lccc}
\hline & $D E(D P)(n=10)$ & Sem DE $(D P)(n=15)$ & Total (DP) $(n=15)$ \\
\hline Idade (anos) & $51.8( \pm 14.74)$ & $31.2( \pm 19.17)$ & $44.9( \pm 18.61)$ \\
Idade de início da ES (anos) & $45.6( \pm 13.23)$ & $22.2( \pm 15.02)$ & $37.8( \pm 17.53)$ \\
Duração da doença (meses) & $74.3( \pm 50.34)$ & $107.2( \pm 80.55)$ & $85.3( \pm 61.16)$ \\
Escore cutâneo & $17.2( \pm 7.28)$ & $13.8( \pm 9.83)$ & $16.1( \pm 8.03)$ \\
\hline
\end{tabular}

$\mathrm{DE}=$ disfunção erétil; $\mathrm{DP}=$ desvio-padrão; $\mathrm{ES}=$ esclerose sistêmica

TABELA 2

Características ClíNicas

\begin{tabular}{lccc}
\hline & $\mathrm{DE}(\%)$ & Sem $\mathrm{DE}(\%)$ & Total $(\%)$ \\
\hline ES difusa & 70 & 40 & 60 \\
\hline ES limitada & 30 & 60 & 40 \\
Envolvimento peniano & 30 & 0 & 20 \\
\hline Raynaud & 100 & 80 & 93 \\
FAN positivo & 56 & 25 & 46 \\
\hline Anti - Scl70 + & 33 & 0 & 20 \\
Hipertensão Pulmonar & 22 & 0 & 14 \\
\hline Fibrose Pulmonar & 56 & 20 & 43 \\
\hline Envolvimento cardíaco & 0 & 0 & 0 \\
\hline Envolvimento esofágico & 67 & 40 & 57 \\
Envolvimento gasrintestinal (outro) & 22 & 0 & 14 \\
Miopatia & 0 & 0 & 0 \\
Envolvimento renal & 0 & 0 & 0 \\
\hline
\end{tabular}

$\overline{\mathrm{DE}}=$ disfunção erétil; $\mathrm{ES}=$ esclerose sistêmica

Cinco homens com DE tinham outras causas de impotência além da ES: insuficiência cardíaca, neuropatia periférica, hipertensão arterial sistêmica, tabagismo e doença obstrutiva arterial periférica. O paciente com neuropatia periférica também fazia uso de uma droga sabidamente associada à DE (amitriptilina). Um destes pacientes referia DE antes do início da ES.

A ES não parece afetar o desejo sexual dos pacientes, uma vez que $66,6 \%$ responderam senti-lo freqüentemente e todos os pacientes graduaram seu desejo como alto ou muito alto.

\section{DISCUSSÃO}

A prevalência da DE varia de acordo com características demográficas, como a média de idade da população. Nos EUA, o Massachusetts Male Aging Study reportou uma prevalência da DE de $34.8 \% \%^{(6)}$. Estudos de populações de pacientes acometidos por outras doenças sabidamente asso-
TABELA 3

Drogas (USO PRÉVio E ATUAL)

\begin{tabular}{lcccc}
\hline & & $\mathrm{DE}(\%)$ & Sem DE $(\%)$ & Total $(\%)$ \\
\hline Penicilamina & Prévio & 20 & 40 & 27 \\
& Atual & 40 & 0 & 27 \\
Ciclofosfamida & Prévio & 30 & 60 & 40 \\
& Atual & 30 & 40 & 33 \\
Asiaticosídeo & Prévio & 30 & 40 & 33 \\
& Atual & 0 & 20 & 7 \\
Bloqueador de canal & Prévio & 20 & 60 & 33 \\
de cálcio & Atual & 60 & 100 & 73 \\
Corticosteróides & Prévio & 20 & 40 & 27 \\
& Atual & 10 & 0 & 7 \\
Pentoxifilina & Prévio & 10 & 0 & 7 \\
& Atual & 20 & 20 & 20 \\
\hline
\end{tabular}

$\mathrm{DE}=$ disfunção erétil

ciadas à DE podem suscitar comparações mais úteis: em diabéticos, a prevalência da DE varia de $40 \%$ a $80 \%$, enquanto em pacientes que sofreram revascularização do miocárdio alcança $57 \%$ e em pacientes com diagnóstico prévio de infarto do miocárdio é de $59 \%{ }^{(1)}$. O tamanho restrito de nossa amostra não permite comparações confiáveis, mas é notável que a prevalência da DE neste grupo seja comparável à de populações de diabéticos e de coronariopatas.

Apesar dos dados disponíveis na literatura sobre a DE na ES serem escassos, esta é uma queixa freqüente entre homens esclerodérmicos, afetando 66,7\% de nosso pacientes. Muito pouco se sabe sobre as causas da DE na ES. Dentre as causas prováveis deve ser enfatizado o envolvimento característico da microcirculação na ES, assim como fibrose do corpo cavernoso e distúrbios psicológicos, freqüentes em pacientes com doenças crônicas. Envolvimento de órgãos-alvo, como insuficiência cardíaca, hipertensão pulmonar e insuficiência renal, também pode contribuir para a gênese da DE. No presente estudo os pacientes com 
DE tinham maior prevalência de envolvimento de órgãosalvo, mas a diferença não alcançou significância estatística. Pacientes esclerodérmicos geralmente utilizam várias drogas, mas apenas um de nossos pacientes utilizava uma droga classicamente relacionada à DE, a amitriptilina.

Não há estudos sobre a prevalência da DE em pacientes com ES. Há, na literatura, apenas alguns relatos de $\operatorname{casos}^{(7-11)}$ e um estudo comparando homens com ES e com artrite reumatóide $^{(12)}$. A prevalência da DE aumenta com a idade, portanto é esperado que a média de idade dos pacientes com DE seja maior. De fato, no presente estudo, os pacientes com DE tendem a ser mais velhos que os sem DE, mas a ocorrência de DE mesmo em jovens e sua alta prevalência na amostra enfatizam que a idade não parece ser um fator causal preponderante para DE na ES. Como a Disciplina de Reumatologia da Unicamp é um centro de referência terciário é esperado que seus pacientes tenham quadros mais graves de ES. É possível que a incidência da DE seja menor em casos mais brandos.

O IIFE pode ser um instrumento útil na avaliação da DE em pacientes com ES, mas a definição corrente da $\mathrm{DE}$ baseada nos critérios de Cappelleri não pode ser aplicada sempre, em razão do grande porcentual de esclerodérmicos que não mantém relações sexuais freqüentes. A natureza crônica da ES, que acarreta incapacidade importante e afeta esteticamente muitos pacientes, pode diminuir seu desejo

\section{REFERÊNCIAS}

1. NIH consensus conference: Impotence. JAMA 270: 83-90, 1993.

2. Rosen RC, Cappelleri JC, Smith MD, Lipsky J, Pena BM: The international index of erectile function (IIEF): a multidimensional scale for assessment of erectile dysfunction. Urology 49: 822-30, 1997.

3. Cappelleri JC, Rosen RC, Smith MD, Mishra A, Osterloh IH: Diagnostic evaluation of the erectile function domain of the international index of erectile function. Urology 54: 346-51, 1999.

4. Ferraz MB, Ciconnelli RM: Tradução e adaptação do índice internacional de função erétil para a língua portuguesa. Rev Bras Med 55: 35-40, 1998.

5. Masi AT, Rodnan GP, Medsger TA Jr, et al: Preliminary criteria for the classification of systemic sclerosis (scleroderma). Arthritis Rheum 23: 581-90, 1980.

6. Feldman HA, Goldstein I, Hatzichristou DG, Krane RJ, McKinley JB: Impotence and its medical and psychological correlates: results of the Massachusetts Male Aging Study. J Urol 151: 54-61, 1994.

7. Mannino M, Marino C: Impotence in scleroderma. Arthritis Rheum 31:148, 1988. sexual ou dificultar o encontro de parceiros. Além disso, tentativas frustradas de obter uma ereção podem fazer com que o paciente evite novas relações sexuais, como exemplificado por alguns de nossos pacientes. O IIFE permite, ainda, graduar a $\mathrm{DE}^{(3)}$, mas não o fizemos no presente estudo em razão do grande número de pacientes que não mantinham relações sexuais regulares. A alta prevalência da DE na ES torna sua pesquisa imperativa, mesmo quando o paciente não o refere espontaneamente. Apesar do grande desconforto, muitos homens com DE evitam contá-lo a qualquer pessoa, incluindo seu médico; o uso de questionários padronizados e auto-administráveis, como o IIFE ou o IIFE5 , sua versão abreviada ${ }^{(13)}$, pode facilitar o diagnóstico.

A melhor estratégia de tratamento da DE em homens com ES ainda deve ser definida, mas estudos recentes de sildenafil na hipertensão pulmonar ${ }^{(14)}$ tornam esta droga um candidato natural. Em estudos futuros de tratamento da DE associada à ES, o IIFE pode ser utilizado para o acompanhamento dos resultados.

Concluindo, grande parte dos homens com ES acompanhados em nosso serviço são acometidos por disfunção erétil. Embora sua causa ainda não esteja clara, a alta prevalência, a ocorrência da DE em pacientes jovens e a concomitância do início da ES e da DE em alguns casos, enfatizam a necessidade de maiores estudos acerca de uma provável relação causal.

8. Rossman B, Zorgniotti AW: Progressive systemic sclerosis (scleroderma) and impotence. Urology 33: 189-92, 1989.

9. Lally EV, Jimenez SA: Erectile failure in systemic sclerosis. N Engl J Med 322: 1398-9, 1990.

10. Lotfi MA, Varga J, Hirsch IH: Erectile dysfunction in systemic sclerosis. Urology 45: 879-81, 1995.

11. Nehra A, Hall SJ, Basile G et al: Systemic sclerosis and impotence: a clinicopathological correlation. J Urol 153:1140-6, 1995.

12. Nowlin NS, Brick JE, Weaver DJ et al: Impotence in scleroderma. Ann Intern Med 104: 794-8, 1986.

13. Rosen RC, Cappelleri JC, Smith MD, Lipsky J, Bena BM: Development and evaluation of an abridged, 5-item version of the International Index of Erectile Function (IIEF-5) as a diagnostic tool for erectile dysfunction. Int $\mathrm{J}$ Impot Res 11: 319-26, 1999.

14. Michelakis E, Tymchak W, Lien D, Webster L, Hashimoto K, Archer S: Oral sildenafil is an effective and specific pulmonary vasodilator in patients with pulmonary arterial hypertension: comparison with inhaled nitric oxide. Circulation 21: 2398-403, 2002. 